\title{
Dynamics of a chain of optically coupled micro droplets
}

\section{Th. Crouzil}

\section{Perrin}

m.perrin@loma.u-bordeauxı.fr
Laboratoire Ondes et Matière d'Aquitaine, Université Bordeaux I, CNRS, 33405 Talence, France

Laboratoire Ondes et Matière d'Aquitaine, Université Bordeaux I, CNRS, 33405 Talence, France

We study a chain of fluid droplets excited by two incoherent laser beams. Such structured object is merely an array of spherical lenses, that can guide a TEMpq optical mode. Taking into account the optical forces exerted by two counterpropagating beams, we show that the droplets can be trapped and the chain auto-organizes in the optical potential. The model takes into account the possible coalescence of several droplets, and shows that the droplet size can increase before they become trapped at stable postitions. For some input beam parameters (beam waist size and position), we have observed dynamic trapping : the droplets experience collective oscillation. Meanwhile, the beam shape evolves periodically in time.

[DOI: http://dx.doi.org/10.2971/jeos.2013.13079]

Keywords: Optofluidics, trapping

\section{INTRODUCTION}

Trapping of neutral Rayleigh particles by a single laser beam has been demonstrated by Ashkin more than 20 years ago [1]. Since then, much work has been devoted to the trapping of nanometric and microscopic particles, and applicative developments have been proposed, concerning on-chip biology [2], microscopy [3], optofluidics [4, 5]. Typically, the trapping potential is created by optical techniques such as, spatial light modulation [6], holographic methods [7], phase contrast methods [8], or by using photonic crystal cavities [9]. In any case, one can expect, at first sight, to find the particles trapped at the bottom of the prescribed potential well, with a significant probability, provided that the incident field is high enough to overcome the thermal energy. But in such simple picture, one assumes a total decoupling between the prescribed potential and the trapped objects. However, experiments have shown that an intense field can induce forces between the trapped particles that modify noticeably their spatial arrangement as well as the shape of the externally imposed potential $[10,11]$. These additional forces are due to the scattering and redistribution of the incident light by the trapped objects. But at first sight, Mie or Rayleigh particles (whose size is close to or smaller than the wavelength) scatter light at a wide solid angle [12], what should lead to a short range interaction between particles. When optical binding takes place, it is a collective response of the scatterers that ensures coupling with a much longer range $[13,14]$. In a situation with two counterpropagating $(\mathrm{CP})$ beams, this can be explained by the refocalisation of the beams [10] by the trapped particles. We transpose such idea at a larger scale, with particle radius, waist size, and inter-particle distance much greater than the wavelength. Then, the diffraction losses can be kept small and almost all the beam energy can be conserved along the chain of trapped objects that would then behave as a wave- guide. In this case, the coupling between particles is expected to be strong, so that a small displacement of one of them can change the force felt by a particle very far from it. Then, one can expect very interesting dynamical properties. Note that breathing and collective oscillations have been observed experimentally and described theoretically for small particles $[10,15]$. Besides, stability and auto-organisation in the transverse plane has been studied for two dimensional structures [16].

In this article, we report on the theoretical study of the dynamics of a unidimensional chain of spherical droplets of fluids (e.g. water in air), that interacts with two CP laser beams. In the first section, we deal with the optical response of a peridodic chain, and describes the optical modes that can be guided in such system. In the second section, we present a simple model used to describe the dynamics, that can handle the collisions that lead to droplet coalescence. The results are presented, that show droplet trapping. In particular, we predict a dynamically trapped state where all the particles undergo collective breathing oscillations.

\section{MODES OF AN OPTICAL CHAIN}

In this section only, we neglect the optical forces, and study the optical response of a chain of droplets to an incident beam. If one considers spherical particles much greater than the wavelength as well as a relatively small index contrast, one can : (i) neglect the backreflection that would otherwise give rise to Fabry Perot interferences between two droplets, (ii) use matrix ray optics (model 1), or a Helmholtz equation (model 2 ) to describe how the field profile changes along the chain. We will 


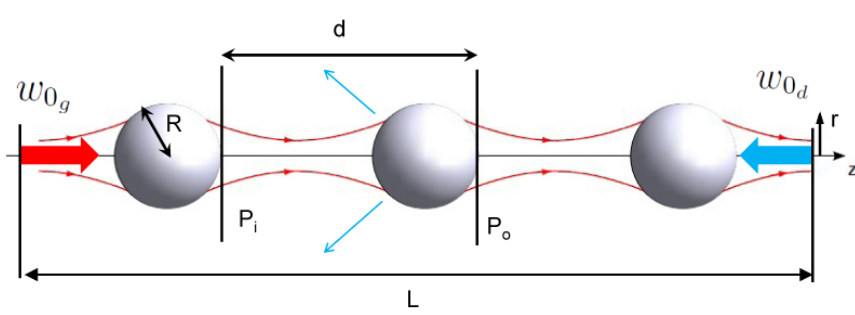

FIC. 1 Scheme of the studied system, with two CP beams whose waists are distant of $L$. The waist size of the beam propagating towards $z>0$ (resp. $z<0$ ) is denoted $w_{0_{g}}$ (resp. $w_{0_{d}}$ ). Note that only three droplets have been sketched for the sake of clarity.

present both model results, and show that they are in good agreement provided that the beam waist on each droplet is significantly smaller than the droplet radius.

Let us consider a uni-dimensional periodic chain made of spherical droplets, of radius $R$, index of refraction $n_{\text {int }}$, plunged into an external medium of index $n_{\text {ext }}$ - see Figure 1 . Optically, they behaves as an ensemble of ball lens of focal length $f=-(R / 2) n_{\text {ext }} /\left(n_{\text {ext }}-n_{\text {int }}\right)$, so that the propagation matrix over one period (of length $d$ ) is :

$$
M=\left(\begin{array}{cc}
1-\frac{d}{n_{\text {ext }} f} & \frac{d}{n_{\text {ext }}} \\
-\frac{1}{f} & 1
\end{array}\right)
$$

This unitary matrix can be diagonalized, so that the operator for $\mathrm{N}$ elementary patterns (propagation over a distance $\mathrm{Nd}$ ) reads :

$$
M_{N}=P^{-1} \cdot\left(\begin{array}{cc}
\Lambda_{+} & 0 \\
0 & \Lambda_{-}
\end{array}\right)^{N} \cdot P,
$$

where $P$ depends on the object plane $P_{o}$ and image plane $P_{i}$ between which one considers the propagation, and $\Lambda_{ \pm}$are the eigenvalues of $M$, that can be written $\Lambda_{ \pm}=\exp ( \pm i \theta)$. Then, a simple calculation gives :

$$
\theta=\arccos \left(1-\frac{d}{2 n_{\text {ext }} f}\right) .
$$

Now, if a Gaussian mode, characterized by its complex radius $q_{0}$ at the center of the first droplet $(\mathrm{z}=\mathrm{d})$ propagates over $\mathrm{N}$ periods, its shape (as described by matrix optics, denoted model 1) becomes $q_{N}=M_{N} \cdot q_{0}$, what can be simply evaluated [17].

Note that, by choosing specific injection condition, one can excite a "Bloch" mode, that will repeat with the period of the chain [18]. This is valid for any value of $\theta$, provided the incident field has a waist size $\omega_{B}$ and is focused at a distance $z_{B}$ of the center of the first lens, with :

$$
\begin{aligned}
\omega_{B} & =\sqrt{\frac{n_{\text {ext }} f \lambda}{\pi}}, \\
z_{B} & =d / 2,
\end{aligned}
$$

then, the waist will be refocused at a point equidistant from 2 neighboring droplets, leading to a periodic field profile similar to the one of Figure 2(a)).

In the general case, when Eqs. (2) are not satisfied, Bloch theorem tells us that the incident field excites a mode whose period is greater than the one of the chain. One can consider that after $\mathrm{N}$ droplets the complex radius of the beam has been dephased of $K 2 \pi$, with $K$ integer. By imposing $\theta=2 \pi K / N$, one gets a condition on the ratio $d / f$ to observe such periodic fields in the chain.

$$
\frac{d}{n_{\text {ext }} f}=2(1-\cos (2 \pi K / N))
$$

As $f$ depends on the droplets radius, one can, by simply vary the distance $d$, excite very different periodic responses in the chain. Some examples are given in Figure 2, where the waist of the Gaussian beam is plotted versus the position in the chain.

This model where only Gaussian beams $\left(\mathrm{TEM}_{00}\right)$ have been considered has the advantage to give simple analytical formulae. However, it is a priori not valid if the waist is comparable to the dropet radius. Therefore, we developed a more accurate, Huygens-Fresnel description of the wavefront evolution. Then, the field at wavelength $\lambda$ in an object plane $P_{o}$ and its image in plane $P_{i}$ are related by an Huygens integral, that can be written in cylindrical coordinates [17]:

$$
\begin{aligned}
\Psi_{i}(r)= & \frac{2 i \pi}{\lambda z} \mathrm{e}^{\left(-\mathrm{i} \frac{\mathrm{kz}}{2}-\mathrm{i} \frac{\mathrm{kr}^{2}}{2 \mathrm{z}}\right)} \\
& \times \int_{0}^{\infty} \Psi_{o}\left(r_{o}\right) \mathrm{e}^{-\mathrm{i}\left(\frac{\mathrm{kr}_{\mathrm{o}}^{2}}{2 \mathrm{z}}+\Delta \Phi\left(\mathrm{r}_{\mathrm{o}}\right)\right)} J_{0}\left(2 \pi \frac{r}{\lambda z} r_{o}\right) r_{o} d r_{o},
\end{aligned}
$$

where $k$ is the wavenumber, $z$ is the distance between the two planes. If the object (resp. image) plane are just before (resp. after) the droplet, $\Delta \Phi\left(r_{o}\right)=\left(n_{i n t}-n_{\text {ext }}\right) \sqrt{R^{2}-r_{o}^{2}}$ is the phase acquired by a ray passing through the droplet at a distance $r_{o}$ from the axis.

Note that this expression takes into account the spherical aberrations and the diffraction that leads to a non-Gaussian profile. Calculations have been carried out using Matlab, and some routines from the OSCAR package [19]. The results are shown in Figure 2, and are in very good agreement with the matrix model, provided that the spot size on the droplet, $\omega$, remains small enough $(\omega \sim R / 5$ in Figure 2$)$.

We have described the beam propagation in a periodic chain of droplets. It comes out that the period of the beam width depends simply on the ratio $d / f$. Besides, for any value of $d / f$, one can excite a mode whose periodicity is exactly the one of the chain. This latter is called a Bloch mode, by analogy to the formalism developed in the physics of periodic media (solid state physics, photonics).

It is not difficult to imagine what would happen if one were to send two intense CP laser beams on the chain. One could arrange so that the same mode profile with the same periodicity is excited by both beams. Then, one can expect to have trapping, with a zero total force exerted on each droplet. However, not all the excited modes would give rise to stable trapping, and some more detailed study is required.

\section{DYNAMICS OF THE CHAIN}

Let us now consider the influence of optical forces. Due to the droplet radius, and the index gradient, optical forces can 


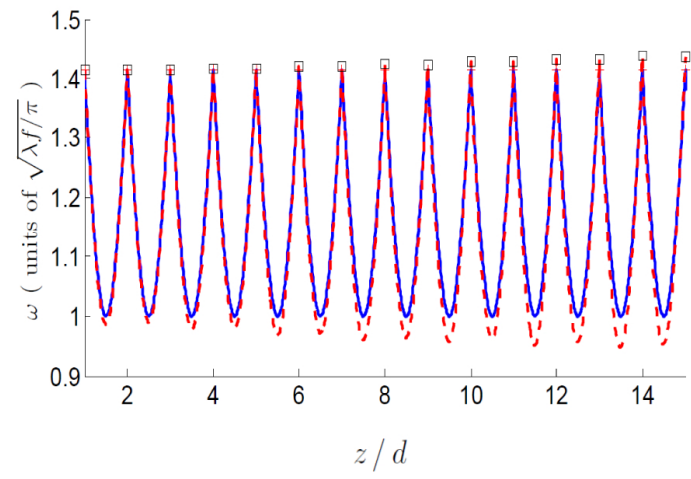

(a) $\mathrm{K}=1, \mathrm{~N}=2$

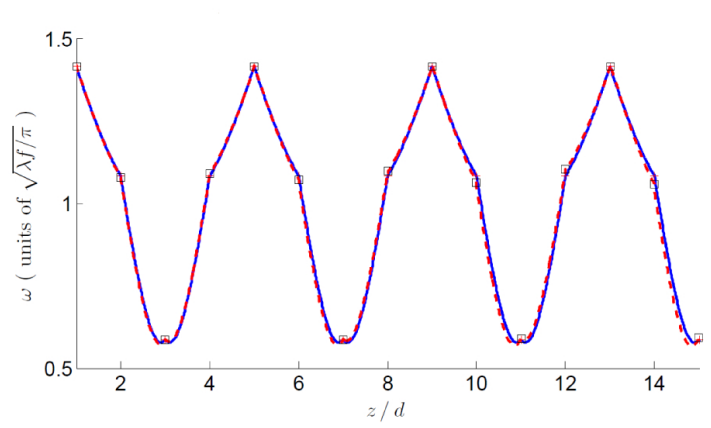

(c) $\mathrm{K}=1, \mathrm{~N}=4$

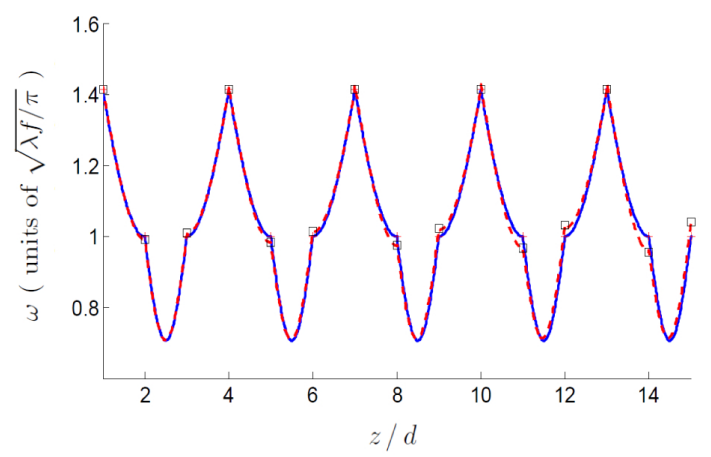

(b) $\mathrm{K}=1, \mathrm{~N}=3$

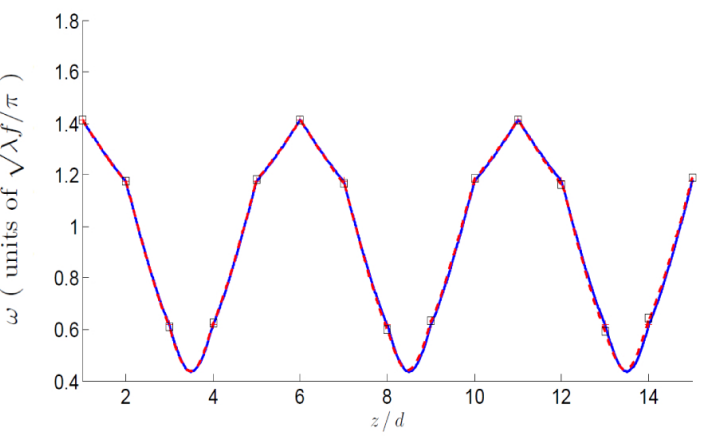

(d) $\mathrm{K}=1, \mathrm{~N}=5$

FIG. 2 Waist profile of the gaussian beam excited for several values of the ratio $d / f$ (see Eq. (3)). The incident waist is $\omega_{0}=R / 5$, with $R=50 \mu \mathrm{m}$. The solid (resp. dahsed) line corresponds to the ray matrix (resp. Huygens) model.

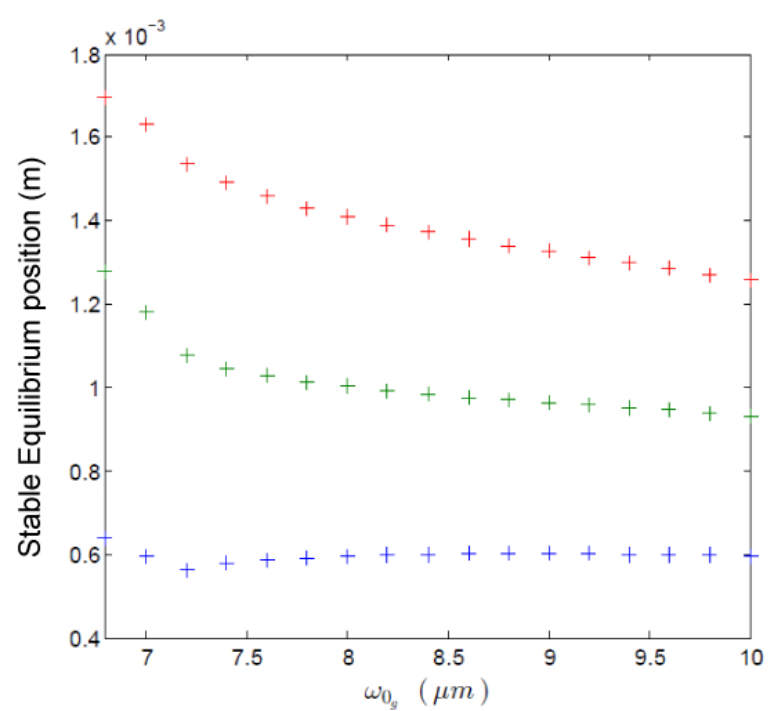

FIC. 3 Stable equilibrium obtained from the fixed point analysis of the equation of motion, for three droplets of $50 \mu \mathrm{m}$ radius in CP beams. Both beams are separated by $L=2 \mathrm{~mm}$. One beam waist is fixed, $w_{0_{d}}=8 \mu \mathrm{m}$ and $w_{0_{g}}$ is varied between $6.75 \mu \mathrm{m}$ and $10 \mu \mathrm{m}$ (see Figure 1). Both beam power is $P=1 \mathrm{~W}$.

be safely computed from ray optics, under the hypothesis of Gaussian beams [20], if the shape remain spherical at all times.

However, one knows that an intense light beam can distort the droplet and thereby introduce large spherical aberrations. Recent works on droplet deformation by gaussian beam can help to evaluate the laser intensity at which this phenomenon occurs [22]. It comes out that the corrections to spherical shape due to the interaction with a beam of power $P$, whose waist $w_{0}$ is smaller than the droplet radius $R$ scale as $\left(R / w_{0}\right)^{2}(P / \zeta)$, where $\zeta$ is the surface tension. In our case, we could expect some small deformation for a power of $1 \mathrm{~W}$ in a spot radius of $w_{o} \sim 10 \mu \mathrm{m}$ impinging on a $50 \mu \mathrm{m}$ radius water droplet, and observe a slight change of the focal length and in the spherical aberrations. However, for the sake of simplicity, we have not considered this effect in the model. Yet, a more precise Huygens-Fresnel description of the beam propagation could be use to compute the forces on a non-spherical surface. In this case one could still use [20] to compute the optical force, but with the correct weight for the intensity of each ray [21].

The equations of motion of each droplets are :

$$
\begin{aligned}
\frac{d z_{i}}{d t} & =v_{i}(t), \\
\frac{d v_{i}}{d t} & =\frac{1}{m}\left[F_{o p t}\left(z_{i}\right)-\gamma v_{i}(t)\right],
\end{aligned}
$$

where $z_{i}, v_{i}$ and $F_{\text {opt }}\left(z_{i}\right)$ are respectively the position, velocity and the longitudinal optical force on the $i^{\text {th }}$ droplet of mass $m$. Note that $F_{o p t}\left(z_{i}\right)$ is a very complex function of the position of all the other droplets, computed from [20]. As we are working at low Reynolds number, the friction coefficient can be expressed [21] as $\gamma=6 \pi \eta R$, where $\eta$ is the dynamic viscosity of the external medium. All the presented result hereafter correspond to water droplets in air. Then, $\eta=1.8 \times 10^{-5}$ Pa.s, $n_{\text {int }}=1.33$.

First, one searches for equilibrium positions. Therefore, we impose the waist size and position of both beams, and search for the $z_{i}$ that satisfy $F_{o p t}\left(z_{i}\right)=0$. Then, the stable states 


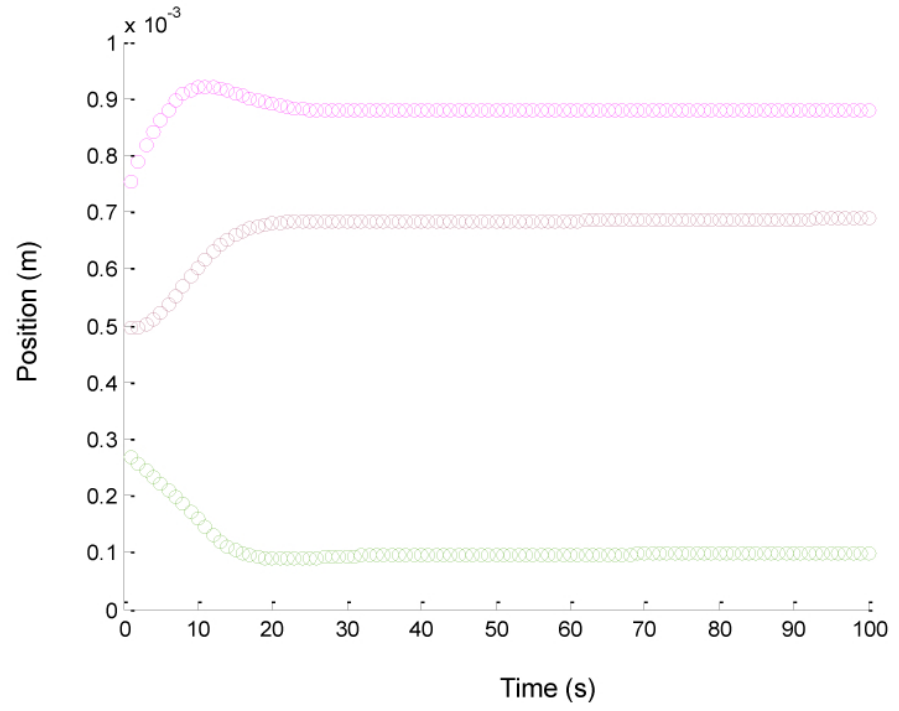

(a) Three droplets chain. $w_{0_{g}}=5 \mu \mathrm{m}, w_{0_{d}}=4 \mu \mathrm{m}, L=1 \mathrm{~mm}$. (see Figure 1)

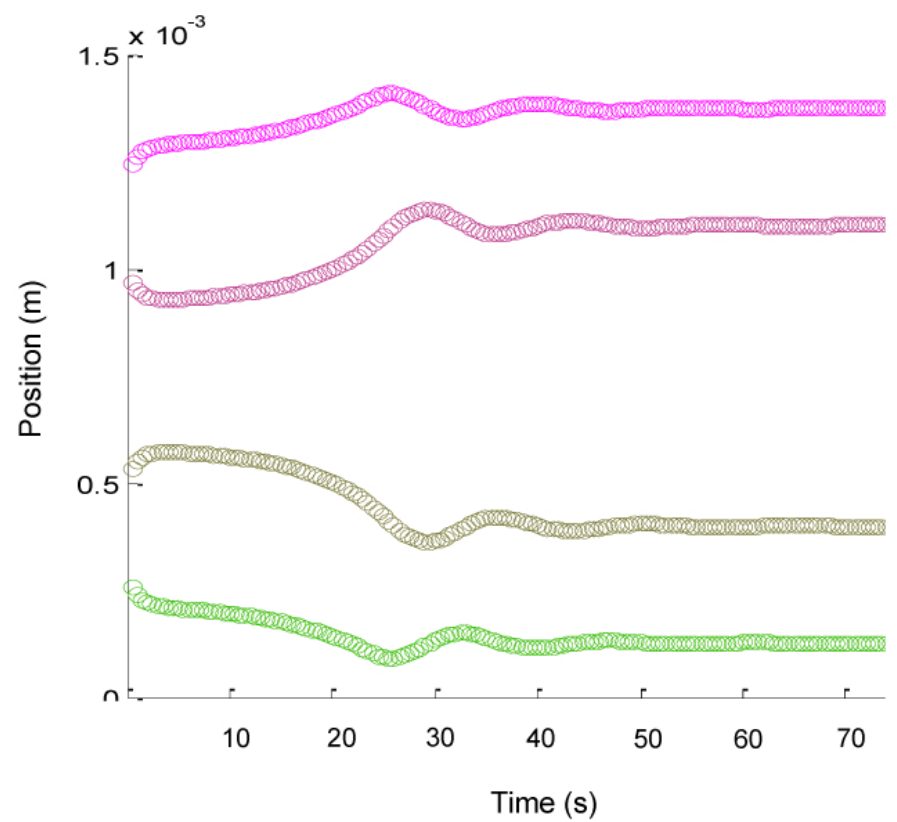

(b) Four droplets chain. $w_{0_{g}}=w_{0_{d}}=5 \mu \mathrm{m} . L=1.5 \mathrm{~mm}$. (see Figure 1)

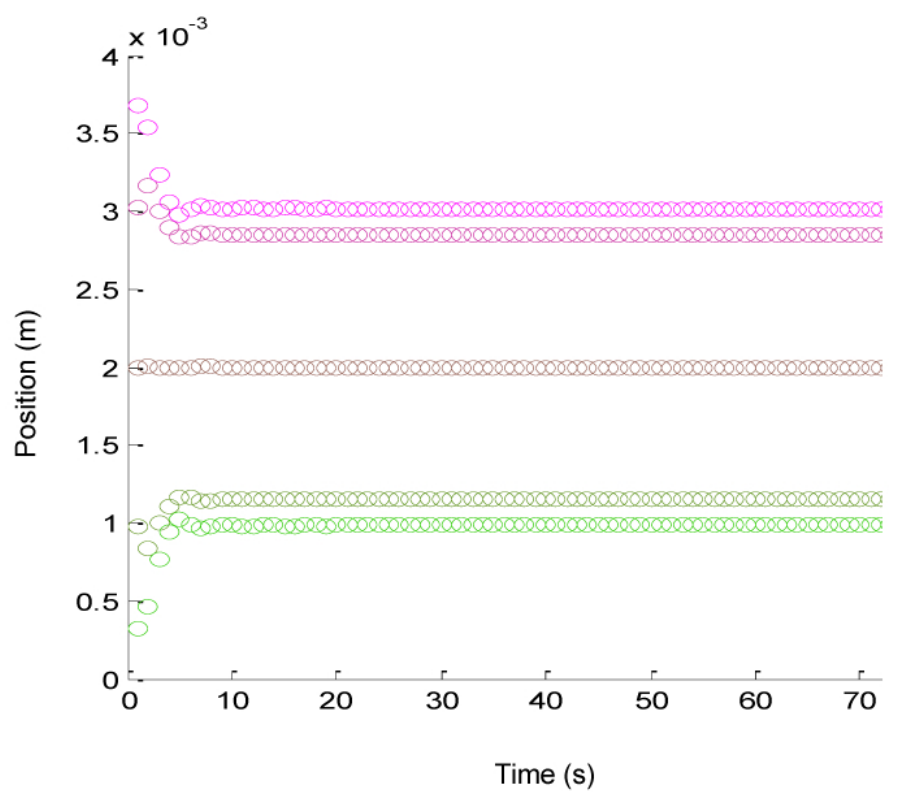

(c) Five droplets chain. $w_{0_{g}}=w_{0_{d}}=10 \mu \mathrm{m} . L=4 \mathrm{~mm}$. (see Figure 1 )

FIC. 4 Time evolution of the chain towards a stable equilibrium. Example of chains of 3,4 and 5 droplets with $R=50 \mu \mathrm{m}$ and $\lambda=514.5 \mathrm{~nm}$. Beam power is $P=1 \mathrm{~W}$. In (b) and (c), the injection conditions are symmetric and the structure is symmetric by respect to $z=L / 2$.In (a) both beams do not have the same waist and the situation is asymmetric.

are found by searching for the eigenvalues of the jacobian of Eq. (5), at the equilibrium positions, that have a negative real part [23]. Figure 3 shows the stable equilibrium positions that we obtained for a short chain, as a function of one incident beam waist (the other being fixed at $8 \mu \mathrm{m}$ ). In general the intensity of both $1 \mathrm{~W}$-power laser beams incident on the droplets is not identical. Nevertheless, the structure can reach a stable and does not move. In the particular case of symmetric injection conditions (when both beams have the same waist, $\left.w_{0_{g}}=w_{0_{d}}=8 \mu \mathrm{m}\right)$, the structure auto-organises exactly in the middle of the space between the two lasers. The droplet in the middle of the chain is stable at $z=L / 2$. In a more general case (asymmetric injection conditions), we noticed that the stable chains are not perfectly periodic.

In order to check that the solutions we obtained indeed cor- 


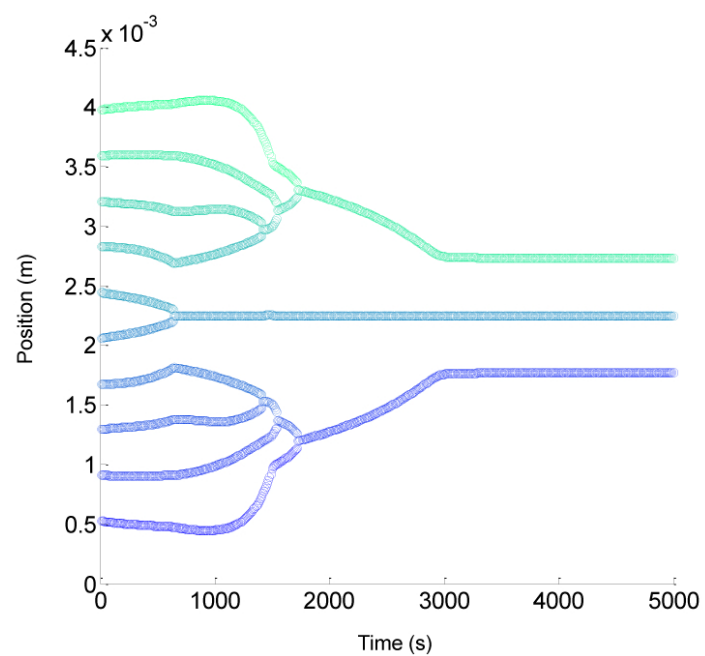

FIG. 5 Time evolution of the chain when coalescence phenomena occurs. $R=50 \mu \mathrm{m}$, $\lambda=514.5 \mathrm{~nm}$ and $L=4.5 \mathrm{~mm}$. The initial chain is periodic and a Bloch mode is excited by symmetric injection condition, see. Eq. (2).

respond to trapped states, we made time simulation, starting from different initial conditions. Then, we observed that the droplets can experience collisions. In order to model what would happen if two or more droplet collide and then coalesce, we developed the following algorithm :

1. One evaluates the mode profile at each droplet position (either from a matrix ray theory or a Huygens integral).

2. Then one computes $F_{o p t}\left(z_{i}\right)$ using [20].

3. Eq. (5) is integrated on a time step $\Delta t$.

4. The process is iterated untill a collision occurs or the simulation is finished.

5. If a collision occurs (between two droplets), one is removed, the radius of the other one is increased, so that the total volume is conserved. Then the dynamics is iterated.

This "molecular dynamics" algorithm has been implemented with Matlab, and after some time, the system generally reaches a steady state, whether collisions occurs or not. Figure 4 shows a typical evolution for short chains. One can see that, varying the injection parameters (the waist size), one can obtain stable trapping after some relaxation oscillation. Note that the time needed to reach the equilibrium depends on the optical force, and is typically of several minutes with an optical power of $\mathrm{P}=1 \mathrm{~W}$. In the case where friction is strong enough so that one can neglect inertia [21], this convergence time scales as $\tau \sim 1 / P$.

If one increases the number of droplets initially present, some of them will be pushed towards one another, and will coalesce. When a collision occurs, the force profile is modified and affects all the other droplets. Eventually, the chain can reach a stable trapped states, but with less droplets than what initially present. Figure 5 shows an example where a 10 droplet system evolves to a 3 droplet one, that is stable in the trap.

This is an example of optically controlled coalescence and trapping with micro droplets that could be observable experimentally.

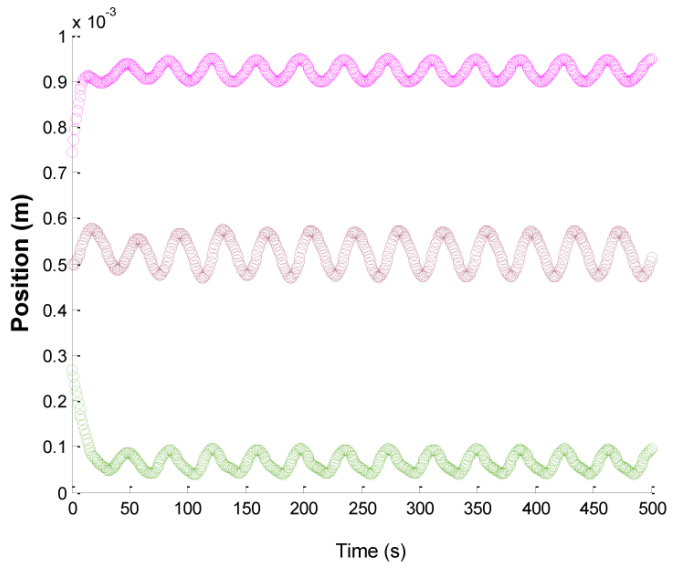

(a) Three droplets oscillating. $w_{0 g}=5 \mu \mathrm{m}, w_{0 d}=6 \mu \mathrm{m}, L=1$ $\mathrm{mm}$

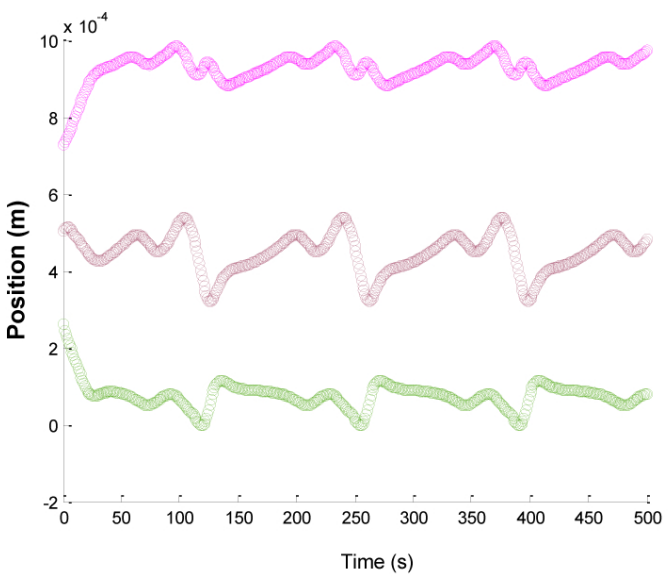

(b) $w_{0 g}=5 \mu \mathrm{m}, w_{0 d}=8 \mu \mathrm{m}, L=1 \mathrm{~mm}$

FIG. 6 Time evolution of a three droplets chain when a breathing state is excited. $R=50 \mu \mathrm{m}$ and $\lambda=514.5 \mathrm{~nm}$. The beam power is $\mathrm{P}=1 \mathrm{~W}$.

For some beam parameters, one observes that no stable fixed point can be reached. However, the droplets trajectories can remain bounded, and the droplets position start to oscillate, see Figure 6. Again, we noticed that the oscillation frequency (in the limit of strong friction) scales linearly with the light power. The fact that a fixed trapped state can destabilize upon changing a parameter (here, the incident beam waists), to become a state of collective oscillation is typical from strongly coupled system, and has been observed elsewhere in the trapping of cold atoms [24]. In opto-fluidics, such oscillations have been obtained with small particles in the transverse plane [16], or in longitudinal binding [15]. But in our work the particles are much larger than the wavelength and their motion modulate the gaussian beam profile in a very simple way. Indeed, we observe a periodic breathing of its waist between each droplet.

\section{CONCLUSION}

We have studied the dynamics of an optical chain for which most of the incident energy is not diffracted away or absorbed, but guided. The strong coupling which is therefore created between the droplets leads to an interesting dynamics. We observed some 'standard' trapped state, where all the droplets 
relax to some fixed position, but also droplet coalescence before trapping. In some cases, the fixed point is unstable, and the system converges to a dynamical trapped state, where all the droplets oscillate periodically in position and velocity. Such dynamics has been observed with solid particles in the Mie regime [16], but not to our knowledge with large droplets that may coalesce. The perspective are mainly theoretical, and are related to the very general problem of spontaneous symmetry breaking and auto-organisation in complex systems. Yet, we have shown that one can control the beam profile through the positions of the droplets. This could be used to make a switch in order to control the beam propagation in the chain. It should be also possible to filter out some modes of a multimode incident beam, using an optofluidic system made of a few droplets actuated by light or by external voltage [25].

\section{ACKNOWLEDGEMENT}

Thomas Crouzil acknowledges support from the Region Aquitaine, allocation number 20091304004.

\section{References}

[1] A. Ashkin, J. M. Dziedzic, J. E Bjorkholm, and S. Chu, "Observation of a single beam gradient force optical trap for dielectric particle," Opt. Lett. 11, 288-290 (1986).

[2] D. J. Odde, and M. J. Renn, "Laser-guided direct writing for applications in biotechnology," TIBTECH 17, 385-389 (1999).

[3] A. Jesacher, C. Maurer, A. Schwaighofer, S. Bernet, and M. RitschMarte, "Full phase and amplitude control of holographic optical tweezers with highefficiency," Opt. Express 16(7), 4479-4486 (2008).

[4] S. J. Hart, and A. V. Terray, "Refractive-index-driven separation of colloidal polymer particles using optical chromatography," Appl. Phys. Lett. 83, 5316 (2003).

[5] T. Kaneta, Y. Ishidzu, N. Mishima, and T. Imasaka, "Theory of optical chromatography," Anal. Chem. 69, 2701-2710 (1997).

[6] J. E. Curtis, B. A. Koss, and D. G. Grier, "Dynamic holographic optical tweezers," Opt. Commun. 207, 169-175 (2002).

[7] E. R. Dufresne, G. C. Spalding, M. T. Dearing, S. A. Sheets, and D. G. Grier, "Computer-generated holographic optical tweezer arrays," Rev. Sci. Instrum. 72, 1810 (2001).

[8] R. L. Eriksen, P. C. Mogensen, and J. Clückstad, "Multiple-beam optical tweezers generated by the generalized phase-contrast method," Opt. Lett. 27(4), 267-269 (2002).
[9] A. Rahmani, and P. Chaumet, "Optical trapping near a photonic crystal," Opt. Express 14, 6353-6358 (2006).

[10] S. A. Tartakova, A. E. Carruthers, and K. Dholakia, "Onedimensional optically bound arrays of microscopic particles," Phys. Rev. Lett., 89, 283901 (2002).

[11] N. K. Metzger, E. M. Wright, W. Sibbett, and K. Dholakia, "Visualization of optical binding of microparticles using a femtosecond fiber optical trap," Opt. Express 14, 3677-3687 (2006).

[12] H. C. van de Hulst, Light Scattering by small particles (Courier Dover Publications, Mineola, 1957).

[13] V. Demergis, and E. L. Florin, “Ultrastrong optical binding of metallic nanoparticles," Nano Lett. 12, 5756-5760 (2012).

[14] J. M. Taylor, L. Y. Wong, C. D. Bain, and G. D. Love, "Emergent properties in optically bound matter," Opt. Express 16(10), 6921-6929 (2008).

[15] M. Kawano, J. T. Blakely, R. Gordon, and D. Sinton, "Theory of dielectric micro-sphere dynamics in a dual-beam optical trap," Opt. Express 16(13), 9306-9317 (2008).

[16] J. Ng, Z. F. Lin, C. T. Chan, and P. Sheng, "Photonic clusters formed by dielectric microspheres: Numerical simulations," Phys. Rev. B 72, 085130 (2005).

[17] A. E. Siegman, Lasers (University Science Books, Sausalito, 1986).

[18] R. G. Wenzel, "Oscillation of gaussian beam parameters in periodic lens waveguides," Opt. Commun. 43, 89-92 (1982).

[19] J. Degallaix, "Oscar a matlab based optical fft code," J. Phys.: Conf. Ser. 228, 012021 (2010).

[20] R. Gussgard, T. Lindmo, and I. Brevik, "Calculation of the trapping force in a strongly focused laser beam," JOSA B 9, 1922-1930 (1992).

[21] A. Isomura, N. Magome, M. I. Kohira, and K. Yoshikawa, "Toward the stable optical trapping of a droplet with counter laser beams under microgravity," Chem. Phys. Lett. 429, 0605165 (2006).

[22] S. Å. Ellingsen, "Theory of microdroplet and microbubble deformation by gaussian laser beam," J. Opt. Soc. Am. B 30(6), 1694-1710 (2013).

[23] S. H. Strogatz, Nonlinear dynamics and Chaos (Perseus Books, Cambridge, 1994).

[24] J. Javaloyes, M. Perrin, and A Politi, "Collective atomic recoil laser as a synchronization transition," Phys. Rev. E 78, 011108 (2008).

[25] A. S. Basu, and Y. B. Gianchandani, "A programmable array for contact-free manipulation of floating droplets on featureless substrates by the modulation of surface tension," J. Microelectromech. S. 18(6), 1163 (2009). 